\title{
Detection of First Order Phase Transitions Using Direct UV Written Integrated Optical Planar Bragg Gratings Intrinsically Defined Within a Silica Micro-Cantilever
}

\author{
C. Holmes, L.G. Carpenter, H.L. Rogers, J.C. Gates, P.G. R. Smith \\ Optoelectronics Research Centre, University of Southampton, SO17 1BJ, UK \\ chh@orc.soton.ac.uk
}

\begin{abstract}
A phase transition sensor is demonstrated using direct UV written Bragg gratings intrinsically defined within a mechanically resonating silica micro-cantilever. Fabrication is uniquely achieved through a combination of micro-machining and direct UV writing. (C2009 Optical Society of America OCIS codes: (130.3120) Integrated Optics Devices; (130.6010) Sensors
\end{abstract}

\section{Introduction}

Cantilever-based sensors have received increasing interest over recent years [1], particularly in the field of mass sensing where such devices offer a completely new type of miniaturised transducer that can monitor small mass variations using surface stress or harmonic oscillations. Resonant frequency monitoring in particular is a reliable principle for achieving quantitative and qualitative measurements. There are numerous biological and chemical applications for such devices. One particular use is the monitoring of first order phase transitions, which could have significant uses in monitoring the progress of chemical reactions or moisture levels in critical storage applications.

The model for a 1D harmonic oscillator can be used to calculate the resonance frequency, $\mathrm{f}$, of a clamped free beam (cantilever), and is dependent upon the force constant, $\mathrm{k}$, and effective mass, $\mathrm{m}^{*}$, expressed as [1].

$$
f=\frac{1}{2 \pi} \sqrt{\frac{k}{m^{*}}}=\frac{n}{2 \pi} \sqrt{\frac{E I}{m L^{3}}}
$$

where $\mathrm{n}$ is a discrete value representing the order of the supported mode, $\mathrm{E}$ is apparent Young's modulus, I is the moment of area and $\mathrm{m}$ is the uniformly distributed mass. A change in these parameters will affect the properties of the cantilever and so shift its resonant frequency. This work presents an integrated optical silica micro-cantilever, with intrinsically defined Bragg gratings to detect first order phase changes. The miniaturised device is fabricated upon a silica-on-silicon platform and offers remote, multiplexed sensing capabilities. The optical nature of the device also has distinct advantages, particularly for applications in flammable environments.

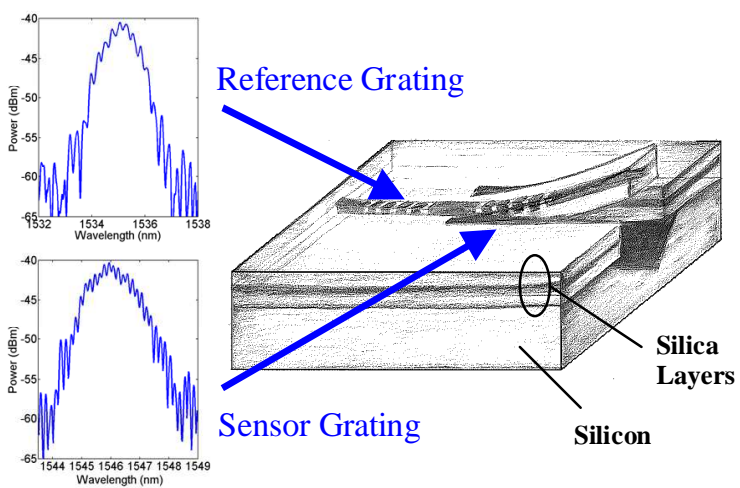

(a)

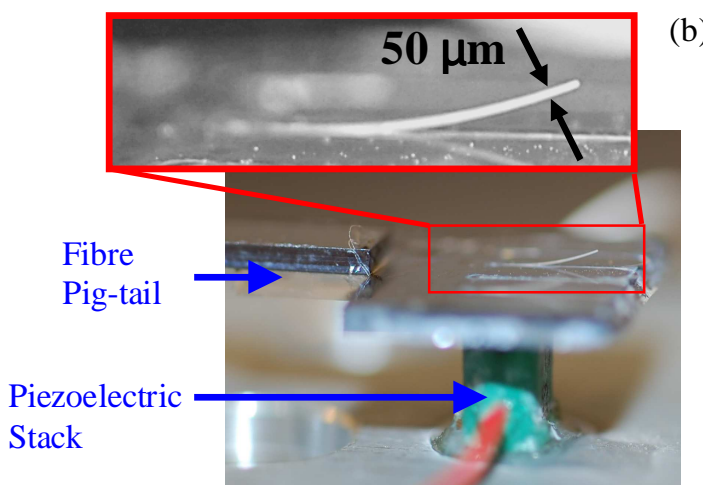

Fig 1. (a) Schematic of the integrated optical cantilever, depicting the locations of the reference and sensing Bragg gratings (b) photograph of the fabricated cantilever (thickness $50 \mu \mathrm{m}$ width $55 \mu \mathrm{m}$ length $4 \mathrm{~mm}$ ) device depicting the secured attachment of a fibre pig-tail and piezoelectric stack

The reported device (illustrated in Figure 1) has a reference Bragg grating located in the bulk of the chip and a sensor Bragg grating located at the point of maximum transduced sensitivity within the cantilever. This is understood theoretically and has been confirmed experimentally to be the location along the cantilever experiencing maximum curvature. The device was fabricated from a silicon-on-silica wafer, using a three stage fabrication process. The first stage used a precision dicing saw to cut the cantilever's outline into the wafers silica layer, 
exposing the underlying silicon. This novel technique allows for rapid prototyping, as it does not require photolithograph and etching steps that can be expensive and time consuming for small device quantities.

The second stage of fabrication defined the Bragg grating elements using a direct UV writing technique [2]. This consisted of two focused, overlapping UV laser beams, which give an inherent interference pattern that is nearcircular and of micron order. Exposure of this intensity pattern into a photosensitive core layer of the silica-onsilicon wafer results in a change in refractive index. If the beam is left on and translated the interference pattern is averaged out, resulting in a channel waveguide. More interestingly, by modulating the writing beam during sample translation channel waveguides with integral index-matched planar Bragg gratings can be fabricated. The combination of these two techniques allows planar Bragg gratings to be inserted into complex UV-written devices in a single processing step.

In the final stage of fabrication the exposed silicon was wet etched using $\mathrm{KOH}$ such to liberate the silica 'outline' from the underlying silicon. The fabricated cantilever reported has a width of $55 \mu \mathrm{m}$ a thickness of $50 \mu \mathrm{m}$ and a length of $4 \mathrm{~mm}$. Interestingly, as can be observed in Figure 1 (b), the cantilever has a buckled form. This is understood to be due to the release of inherent stress caused by the silica-on-silicon wafer fabrication technique.

\section{Results}

Both stress and resonant frequency measurements were taken using the cantilever. To monitor the Bragg gratings as a function of induced stress an Optical Spectrum Analyser (OSA) and broadband light source were used. By cooling the cantilever to $-50{ }^{\circ} \mathrm{C}$ and allowing it to gain temperature over three hours ice accumulation was observed, illustrated in Figure 2 (a). At $0{ }^{\circ} \mathrm{C}$ the melting phase transition occurred and was observed by the spectral response of the sensor Bragg grating. The shift resulted in a reduction of stress as a result of the accumulated ice melting. To monitor the accumulation of small levels of condensation, the cantilever was resonated using a driven piezoelectric stack, situated as in Figure 1 (b). A tuneable laser was aligned spectrally to the FWHM shoulder of the Bragg grating. The reflected power was in turn fed into a photodiode which was referenced against the input signal using a lock-in amplifier, from which phase and amplitude information was monitored as illustrated in Figure 2 (b).

(a)

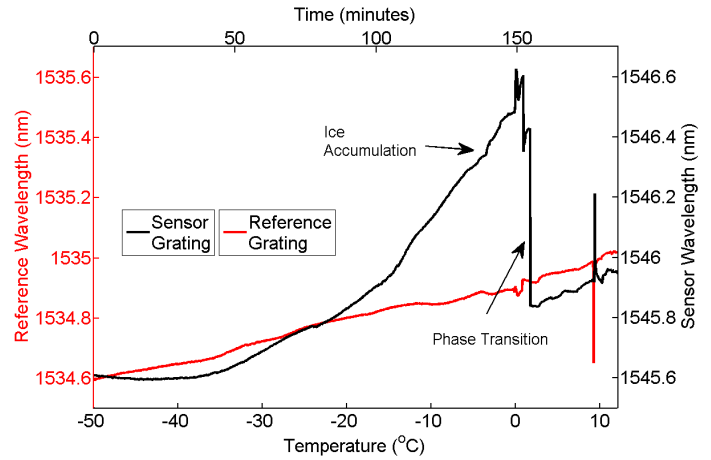

(b)
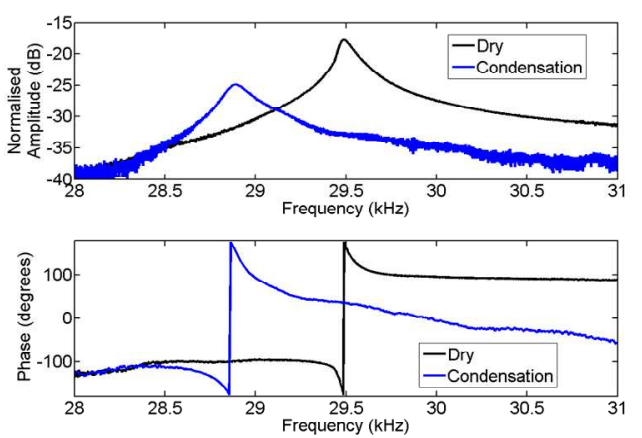

Fig. 2. (a) The spectral shift variations of the reference and sensor Bragg gratings, as a result of ice formation and solid to a liquid phase transition (b) The shift in resonance frequency of the cantilever as a result the presence of condensation

The natural frequency of the cantilever is $29.48 \mathrm{kHz}$, which is close to the approximated theoretical value of 29.11 $\mathrm{kHz}$, calculated from Equation 1. The Q-factor of the measured resonance is 209 at atmospheric pressure. Upon deliberately causing condensation the resonant frequency shifts to $28.88 \mathrm{kHz}$. From Equation 1, it can be inferred that such a shift corresponds to a distributed mass increase of $50 \mu \mathrm{g}$, or a $36 \mu \mathrm{m}$ thick layer of condensed water.

\section{Conclusion}

We have demonstrated for the first time a direct UV written Bragg grating mass sensor, based upon cantilever operation. The sensor has demonstrated the detection of first order phase changes through monitoring inherent stress and natural resonance shifts. We shall report on the fabrication limitations and the use of enhancement layers for improved response measurements for several chemical species.

[1] C. Ziegler, "Cantilever-based biosensors," Anal Bioanal Chem 379, 946-959 (2004)

[2] G. D. Emmerson S. P. Watts et al. "Fabrication of directly UV-written channel waveguides with simultaneously defined integral Bragg Gratings," Electronics Letters 38, 1531-1532, (2002) 